\title{
Bony Erosion with Mucoid Degeneration of the Anterior Cruciate Ligament
}

\author{
Yusuke Ueda, $\mathrm{MD}^{1}$ Kazuo Saita, MD, $\mathrm{PhD}^{1}$ Takehiko Yamaguchi, MD, PhD ${ }^{2}$ Hitoshi Sekiya, MD, $\mathrm{PhD}^{3}$ \\ Toru Akiyama, MD, $\mathrm{PhD}^{1}$
}

${ }^{1}$ Department of Orthopaedic Surgery, Saitama Medical Center, Jichi

Address for correspondence Toru Akiyama, MD, PhD, Department of Medical University, School of Medicine, Saitama, Japan Orthopaedic Surgery, Saitama Medical Center, Jichi Medical University,

2 Department of Pathology, Dokkyo Medical University Koshigaya

Hospital, Koshigaya-shi, Saitama, Japan School of Medicine,1-847 Amanuma, Omiya, Saitama 3308503, Japan

${ }^{3}$ Department of Orthopaedic Surgery, Jichi Medical University,

Shimotsuke, Tochigi, Japan

J Knee Surg Rep 2015;1:25-30.

\begin{abstract}
Keywords

- knee

- anterior cruciate ligament

- mucoid degeneration

- bone erosion
\end{abstract}

The first reported case of mucoid degeneration of the anterior cruciate ligament (MDACL) was provided by Kumar et al in 1999. ${ }^{1}$ The pathogenesis is still unclear, but this condition is thought to be secondary to ACL degeneration. A 2004 study reported the prevalence of MDACL as $0.043 \%,{ }^{2}$ but a 2008 study found a prevalence of $5.3 \%{ }^{3}$ Although MDACL is not as rare as previously thought, it is not still well known by physicians and is probably underdiagnosed or misdiagnosed as an ACL tear. More accurate data on MDACL are needed. MDACL with erosion of adjacent bone is extremely rare; only a few cases have been reported to date. ${ }^{1,4,5}$ We treated two patients with MDACL, one with an eroded lesion in the lateral femoral condyle and one with tibial insertion of the ACL. We report these two cases and discuss their clinical features with reference to previous MDACL reports, focusing on bone erosion.

\section{Patients and Methods}

The two patients were male, aged 38 and 34 years. Their presenting complaints were knee pain, inability to flex beyond 125 and 140 degrees, respectively, and difficulty climbing up and down stairs or walking short distances (less than $500 \mathrm{~m}$ ). The knee pain was not limited to specific points, spreading out to the whole knee in both the cases. Both patients' preoperative received

November 11, 2013

accepted after revision

September 27, 2014

published online

January 12, 2015
DOI http://dx.doi.org/

10.1055/s-0034-1399759. ISSN 2326-2729.
Copyright $\odot 2015$ by Thieme Medical Publishers, Inc., 333 Seventh Avenue, New York, NY 10001, USA. Tel: +1(212) 584-4662.
License terms

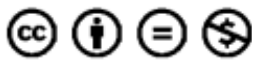


request was just pain relief for activities of daily living, not returning to hard sports. One patient's job was working on a lathe; the other patient worked in mill management. We evaluated the preoperative and postoperative functional level of each patient using the Tegner Lysholm Knee Scoring Scale. ${ }^{6,7}$ Their preoperative scores were 85 (grading; good: 5-5-2515-15-10-6-4) for patients 1 and 72 (grading; fair: 3-2-2515-15-2-6-4) for patient 2 (- Table 1 ).

In both the cases, there was no contralateral knee pain. The symptoms were progressive and not preceded by any significant trauma. No significant swelling or painful points were noted in either patient. A manual knee laxity test (the Lachman test) was performed pre-, intra-, and postoperatively for both the cases, and we also evaluated the anterior laxity at manual maximum stress using a KT-1000 arthrometer (MEDmetric Corp., San Diego, CA $)^{8,9}$ pre- and postoperatively for patient 1 and only postoperatively for patient 2 . Contralateral knee exams showed normal data.

MRI was performed before the patients' arthroscopies. The magnetic resonance imaging (MRIs) showed thickened ACLs with bony erosion of the lateral femoral condyle in patient 1 and tibial insertion of the ACL in patient 2 (-Figs. 1 and 2 ).

Both ACLs had low and homogeneous signal intensity on T1-weighted images and homogeneous high signal intensity on T2-weighted images. In patient 2, not only the peripheral part of the hypertrophic ACL but also the bony eroded lesion was enhanced by gadolinium administration on T1-weighted images (-Fig. 2). The menisci and posterior cruciate ligament (PCL) were normal. We suspected MDACL-induced bony erosion at the site of $\mathrm{ACL}$ insertion. Both patients then underwent arthroscopic surgery.

Under arthroscopy, there were no meniscal pathological findings medially or laterally. The ACL appeared homogenous, bulbous, and hypertrophied in the region of the posterolateral bundle (PLB) of the ACL in patient 1 and in the region of both the PLB and anteromedial bundle (AMB) in patient 2. The PCL was intact in each patient. The ACL fibers were interspersed with white or yellowish tissue. The arthroscopy revealed the impingement of the PLB on the posterolateral part of the knee in both the patients. The hypertrophied tissues were debrided to avoid impingement of the PLB into the posterior joint space, and nearly half of the native ACL was removed in each patient.

We did not reconstruct the ACL in either patient, because their intraoperative Lachman tests did not show any instability derived from ACL insufficiency. Care was taken to confirm that each remaining ACL had an intact attachment to the femoral condyle and did not impinge on the roof or lateral wall of the notch. In addition, the arthroscopic findings of both the patients did not demonstrate anterior tibial insertion osteophytes, posterior capsular retraction, or notch stenosis. Thus, we decided not to perform notchplasty for

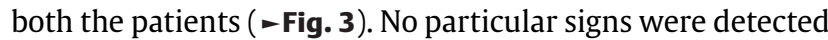
on the wall of the condyle. No other significant pathology was seen in the knees.

\section{Results}

Two weeks after their operations, both the patients achieved full active and passive ranges of motion without pain. At the latest follow-up point, at 2 years and 8 months for patient 1 and 2 years and 1 month for patient 2 , both the patients had a full range of pain-free motion without any impediments to daily living. Neither of the patients had any symptoms of instability. The Lachman test was negative in both knees of the two patients. In patient 1, the KT-1000 test demonstrated 1-mm laxity preoperatively and 3-mm laxity of the operated knee compared with the intact knee 1 year after the operation. For patient 2, the KT-1000 test 1 year and 4 months after the operation demonstrated 1-mm laxity of the intact knee compared with the operated knee. The histology of both cases revealed fragments of ligament tissue exhibiting extensive myxoid changes and small mucus pools in addition to irregular cracks, fibrillation, and focal development of granulation. The overall findings of both cases were consistent with mucoid degeneration of the ligament (-Fig. 4).

Full weight bearing was allowed just after the operation for both the patients in light of the eroded bone lesion localized in the non-weight-bearing part of the knee, and neither patient complained of knee pain induced by weight bearing before or after the operation.

Table 1 The patients' Tegner Lysholm Knee Scoring Scale scores

\begin{tabular}{|l|l|l|l|l|}
\hline & Preoperative patient 1 & Postoperative patient 1 & Preoperative patient 2 & Postoperative patient 2 \\
\hline Limp & 5 & 5 & 3 & 5 \\
\hline Support & 5 & 5 & 2 & 5 \\
\hline Pain & 25 & 25 & 25 & 25 \\
\hline Instability & 15 & 25 & 15 & 25 \\
\hline Locking & 15 & 15 & 15 & 15 \\
\hline Swelling & 10 & 10 & 2 & 10 \\
\hline Stair-climbing & 6 & 10 & 6 & 10 \\
\hline Squatting & 4 & 5 & 4 & 5 \\
\hline Total & 85 & 100 & 72 & 100 \\
\hline
\end{tabular}



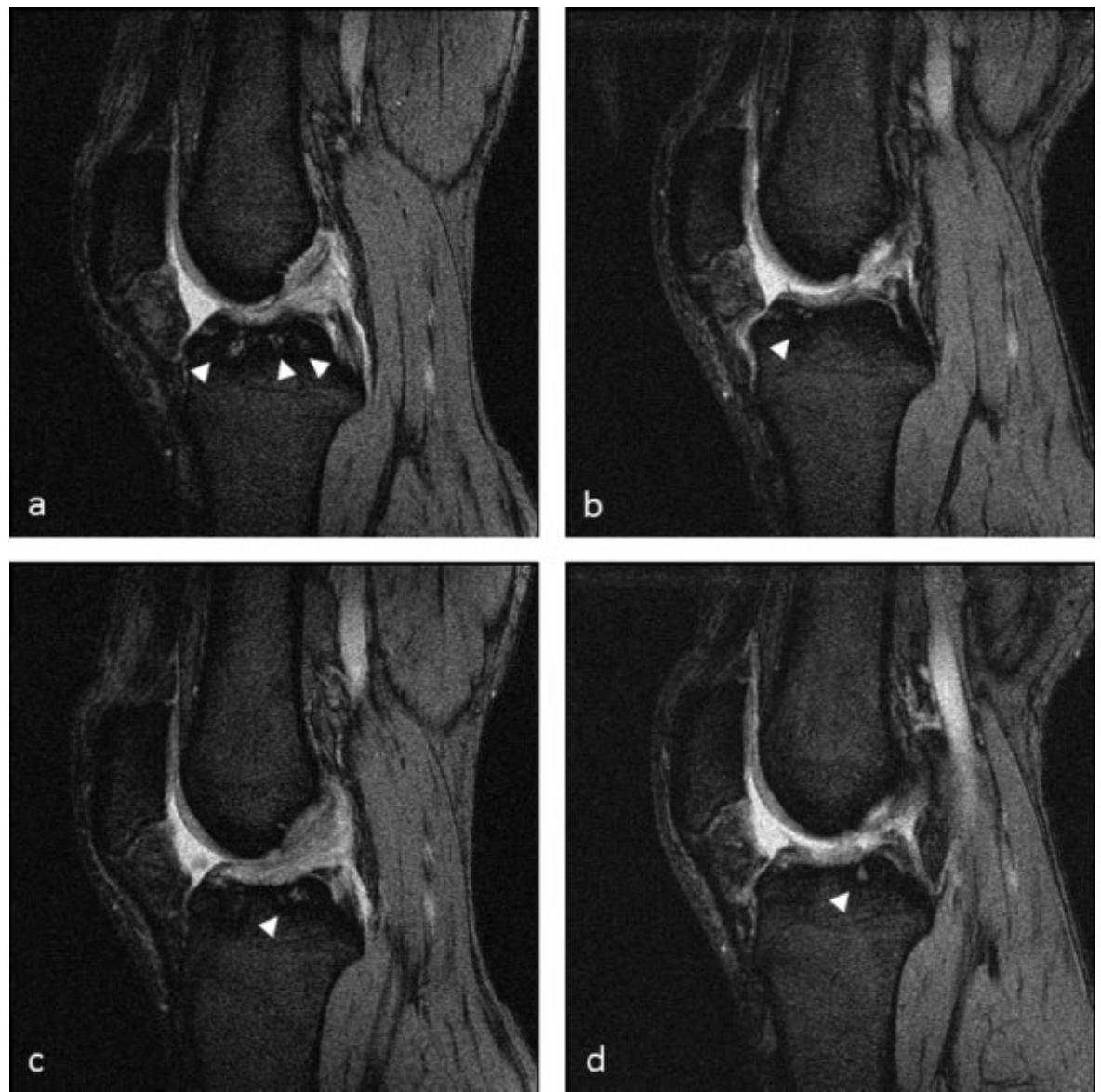

Fig. 1 Patient 1: Magnetic resonance imaging T2-weighted fat suppression images before the operation (a, c) and 1 year after the operation (b, d). Hypertrophic anterior cruciate ligament and bony erosions demonstrated homogeneous high signal intensity on T2-weighted images. Arrowheads indicate bony erosion. The number and volume of eroded lesions after the operation were reduced compared with before the operation.

The Lachman test for both patients did not demonstrate severe joint laxity. The postoperative X-ray examinations for both patients did not show bony deformity. Thus, we expected the patients to be able to return to their jobs depending on their pain levels, with monthly X-ray examinations and a computed tomography (CT) or MRI every 6 months. Both the patients returned to their jobs 1 month after their operations. The knee pain and knee instability have not returned in either patient as of 2 years and 8 months and 2 years and 1 month after their respective operations. For both patients, the postoperative Tegner Lysholm Knee Scoring Scale values were 100 (grading: excellent; 5-5-25-2515-10-10-5) (-Table 1).

In patient 1,1 year after the operation, the follow-up MRI showed reduced volume and a reduced number of eroded bone lesions ( - Fig. 1). For patient 2, 1 year and 4 months after the operation, the follow-up CT and MRI demonstrated the regeneration of the defective cortical bone adjacent to the eroded lesion (-Fig. $\mathbf{5}$ ).

\section{Discussion}

A series of patients with MDACL was recently described in detail, including the MRI findings and differential diagnoses. ${ }^{10,11}$ Here, we present an unusual finding of mucoid degeneration involving the adjacent femoral condyle. Although MDACL had been considered rare, recent publications have shown that it is more frequent than previously thought. The data reported by Bergin et $\mathrm{al}^{2}$ were derived from a retrospective study of 4,221 consecutive knees referred for MRI. They found that only 18 patients $(0.043 \%)$ had mucoid degeneration. ${ }^{2}$ However, Salvati et al reported that their analysis of 1,215 knee MRI examinations identified 64 patients (5.3\%) with ACL mucoid metaplastic degeneration. ${ }^{3}$

Most commonly, patients present with deep posterior knee pain and limited knee flexion. ${ }^{12}$ However, our two patients presented with knee pain the exact location of which could not be identified. In addition, posterior knee pain and the limitation of knee flexion associated with a diffuse thickened ACL are not sufficient to establish an accurate diagnosis of mucoid degeneration.

Lintz et al reported an MDACL population of 19 men and 8 women with a mean age of 49.3 years. ${ }^{12}$ The largest study of MDACL involved a population consisting of 36 men and 38 women with a mean age of 42 years. ${ }^{13}$ The MDACL patients were predominantly middle-aged, and the population showed a slight male predominance. The diagnosis of MDACL was initiated with MRI findings of a hypertrophic ACL demonstrating a high-intensity intermingled mucoid component 

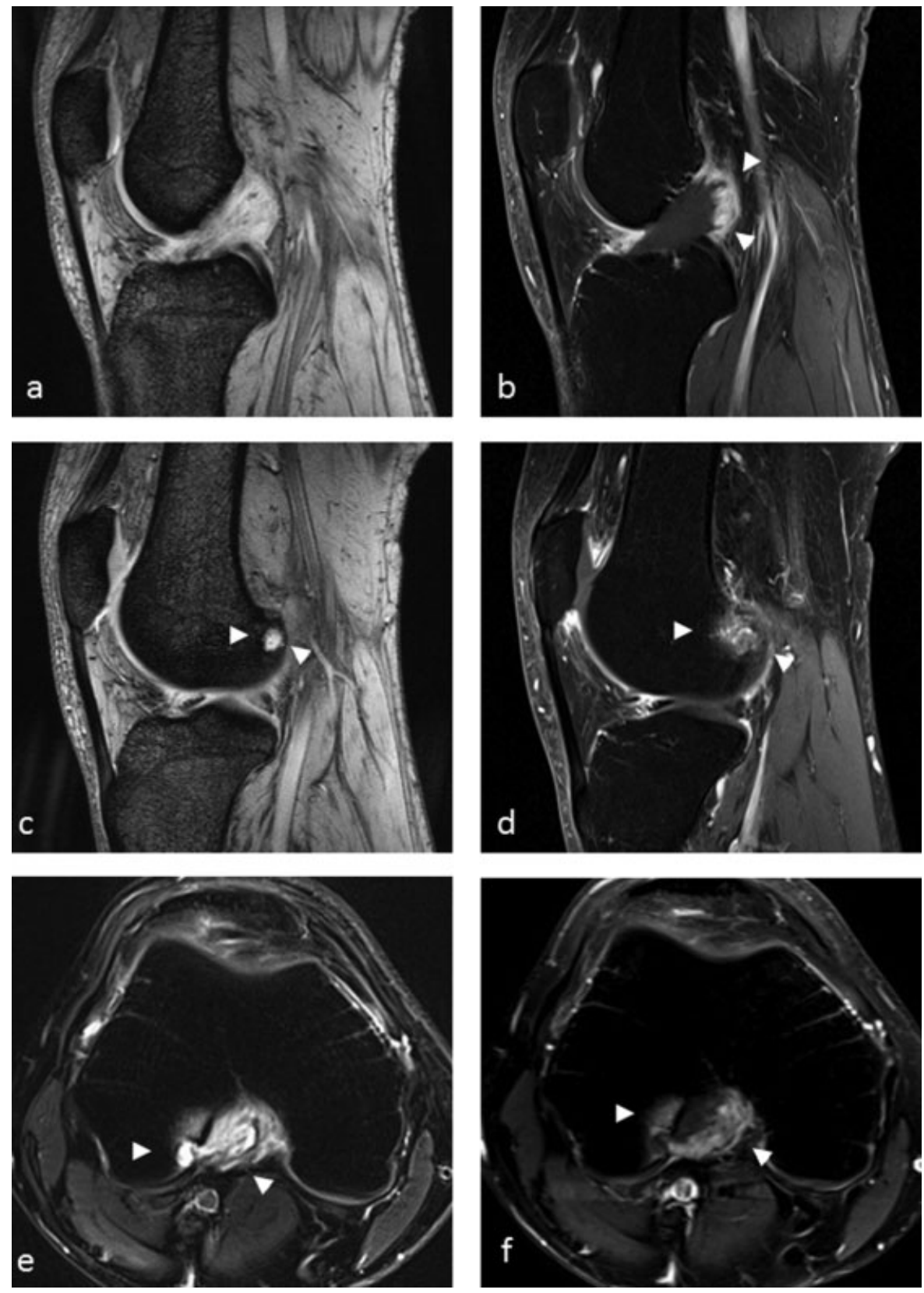

Fig. 2 Patient 2: Magnetic resonance imaging T2-weighted fat suppression images (a, c, e) and T1-weighted gadolinium-enhanced images (b, d, f). Arrowheads indicate bony erosion. Only the peripheral area of the degenerated anterior cruciate ligament and bony erosion were enhanced on T1-weighted gadolinium-enhanced images.

with uninterrupted ligament bundles in T2-weighted images. ${ }^{10}$

Histologically, mucoid lesions contain a brown or yellow basophilic substance that has a high density of glycosaminoglycans such as hyaluronic acids. ${ }^{10}$ The pathological cause of MDACL is still unclear. ${ }^{12}$ In some patients, an initiating knee injury was reported. In these patients, mucoid lesions are thought to arise from an injury or chronic impingement of the ACL. However, several reports including ours described MDACL patients without any known injury. ${ }^{13,14}$

MDACL is frequently found in osteoarthritic knees. In osteoarthritic knees, continuous impingement between the $\mathrm{ACL}$ and an osteoarthritic intercondylar notch tightened by a rim of prominent osteophytes triggers the formation of MDACL. Narvekar and Gajjar resolved the impingement of the ligament on the roof and the lateral wall of the notch via a notchplasty. ${ }^{13}$ In another study, MRI demonstrated that knees with MDACL possessed a narrower notch, a steeper notch angle, and a smaller notch area compared with controls. ${ }^{15}$ These reports suggest that continuous impingement of tighter notches on the ACL results in repeated microtrauma and the formation of MDACL.

The treatment of MDACL is usually arthroscopic resection of part or all of the ACL, aiming for the debridement of the afflicted portion of the ACL, sometimes with notchplasty. ${ }^{13,16}$ 


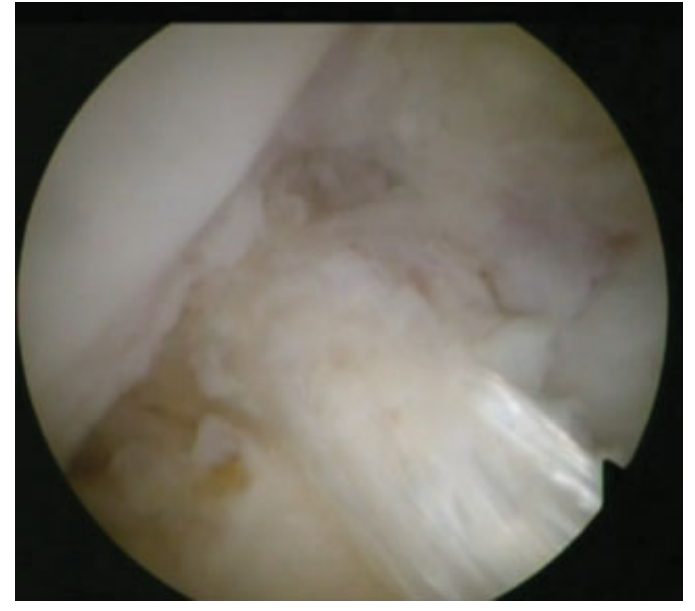

Fig. 3 Intraoperative finding of patient 2 demonstrated that notchplasty was not required to avoid impingement of the anterior cruciate ligament.

In our patients, we debrided only the PLB in patient 1 and both the complete PLB and a part of the AMB in patient 2 to avoid impingement at the posterolateral part of the knee joint. The knee pain was relieved quickly and continuously at least for 2 years without any symptoms. Thus, the resected

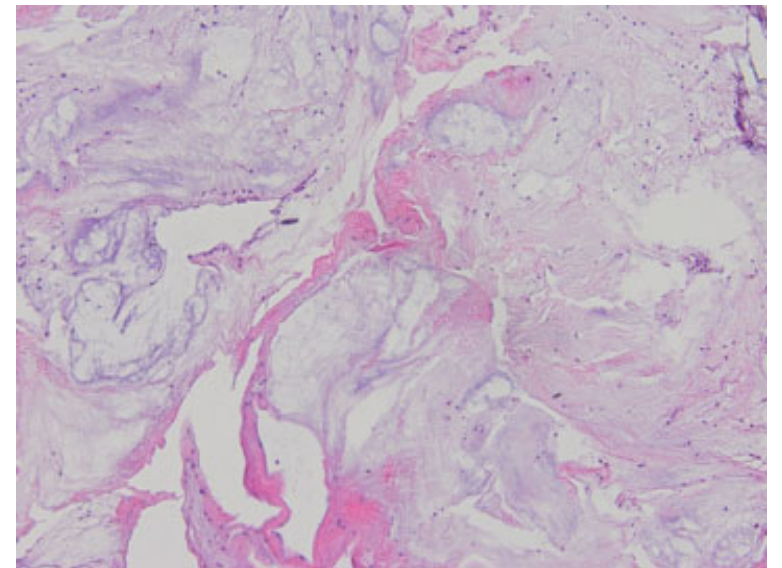

Fig. 4 Histology photo of patient 2. Cracked ligament tissue exhibiting extensive mucoid degeneration.

part and volume must be determined by intraoperative findings.

Erosion of adjacent bone associated with MDACL is extremely rare. To the best of our knowledge, only a single case of this pathological finding has been reported to date. ${ }^{5}$ Canine
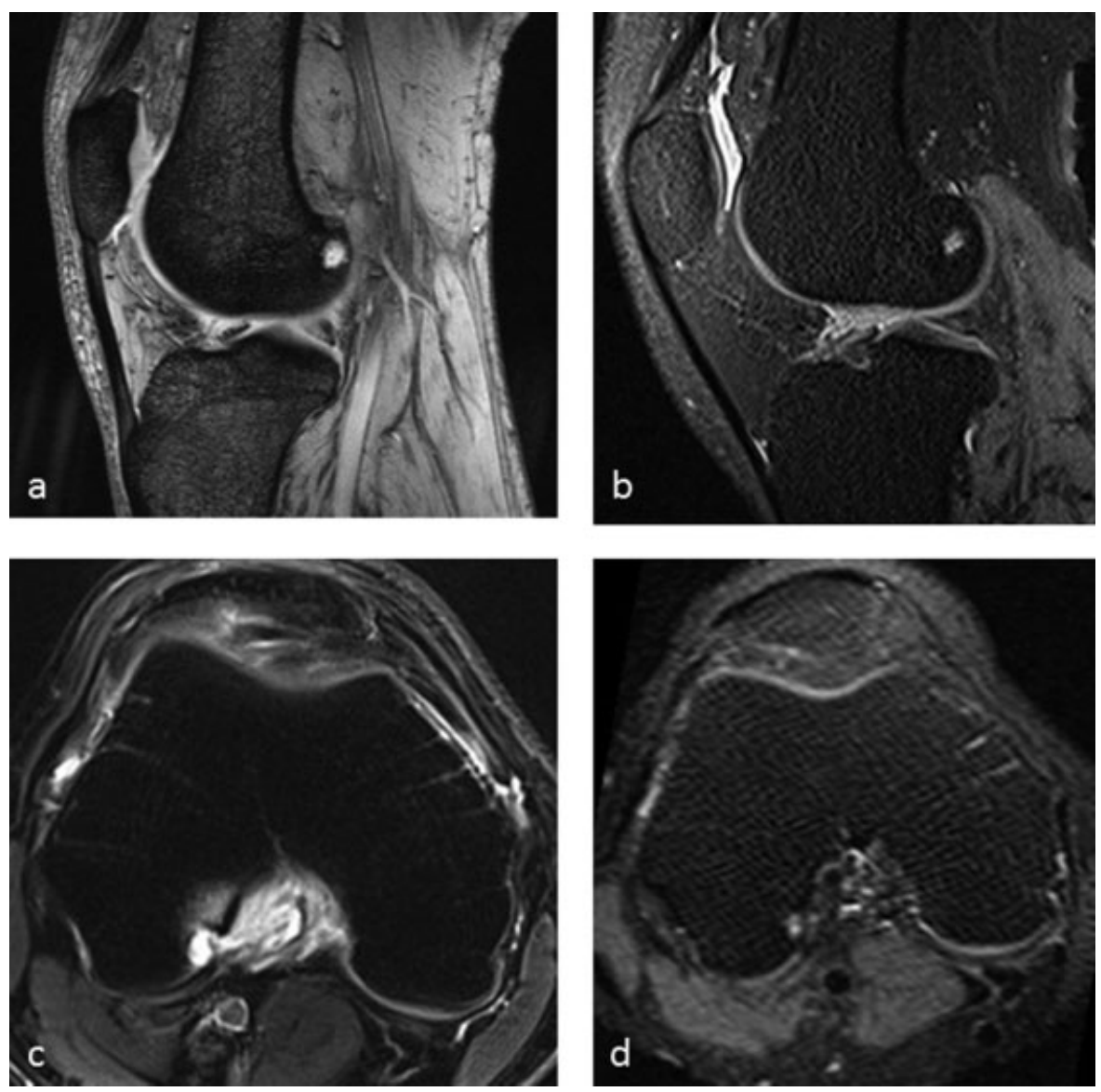

Fig. 5 Patient 2: Magnetic resonance imaging (MRI) T2-weighted fat suppression images (a, c) before the operation and (b, d) 1 year and 4 months after the operation. The follow-up MRI demonstrated the regeneration of cortical bone adjacent to the bony erosion. 
progressive ACL degeneration was induced by cathepsin $\mathrm{K}$ and tartrate-resistant acid phosphatase (TRAP). ${ }^{17}$ Similar changes in human ACLs implicating the same enzymes have been demonstrated. ${ }^{18}$ Cathepsin $\mathrm{K}$ and TRAP are osteoclast-specific enzymes. ${ }^{19-21}$ These reports suggest that the development of MDACL is related to osteoclast induction, directly or indirectly. Osteoclasts are multinucleated giant cells primarily responsible for bone resorption. ${ }^{19} \mathrm{~A}$ careful reading of the existing literature on MDACL reveals several figures that show or suggest possible collateral osseous lesions. ${ }^{2,11,13}$ Intraosseous lesions may not be uncommon collateral lesions in MDACL.

We treated our two patients with bony erosion by debriding only the ACL lesion, not the bony lesion. In each patient, the intractable knee pain had vanished at 2 weeks after this ACL debridement. In both the patients, the bony erosion lesions were reduced or regenerated after the ACL debridement. These outcomes suggest that the level of pain reliefnot healing the bony erosion lesion or joint laxity-is the optimum indicator of the success of treatment.

In addition, our patients' bony lesions did not affect their weight-bearing level or rehabilitation. Thus, the bony lesion is likely not the cause of the knee pain, which is possibly triggered by MDACL-induced osteoclasts. Although the pathological cause remains unclear, our new findings are clues that may help reveal the pathogenesis of MDACL in further clinical research.

\section{References}

1 Kumar A, Bickerstaff DR, Grimwood JS, Suvarna SK. Mucoid cystic degeneration of the cruciate ligament. J Bone Joint Surg Br 1999; 81(2):304-305

2 Bergin D, Morrison WB, Carrino JA, Nallamshetty SN, Bartolozzi AR. Anterior cruciate ligament ganglia and mucoid degeneration: coexistence and clinical correlation. AJR Am J Roentgenol 2004; 182(5):1283-1287

3 Salvati F, Rossi F, Limbucci N, Pistoia ML, Barile A, Masciocchi C. Mucoid metaplastic-degeneration of anterior cruciate ligament. J Sports Med Phys Fitness 2008;48(4):483-487

4 Motmans R, Verheyden F. Mucoid degeneration of the anterior cruciate ligament. Knee Surg Sports Traumatol Arthrosc 2009; 17(7):737-740

5 Melloni P, Valls R, Yuguero M, Sáez A. Mucoid degeneration of the anterior cruciate ligament with erosion of the lateral femoral condyle. Skeletal Radiol 2004;33(6):359-362

6 Tegner Y, Lysholm J. Rating systems in the evaluation of knee ligament injuries. Clin Orthop Relat Res 1985;(198):43-49
7 Mitsou A, Vallianatos P, Piskopakis N, Maheras S. Anterior cruciate ligament reconstruction by over-the-top repair combined with popliteus tendon plasty. J Bone Joint Surg Br 1990;72(3):398-404

8 Hoshino Y, Kuroda R, Nagamune K, et al. In vivo measurement of the pivot-shift test in the anterior cruciate ligament-deficient knee using an electromagnetic device. Am J Sports Med 2007;35(7): 1098-1104

9 Daniel DM, Stone ML, Sachs R, Malcom L. Instrumented measurement of anterior knee laxity in patients with acute anterior cruciate ligament disruption. Am J Sports Med 1985;13(6): 401-407

10 Lintz F, Pujol N, Boisrenoult P, Bargoin K, Beaufils P, Dejour D. Anterior cruciate ligament mucoid degeneration: a review of the literature and management guidelines. Knee Surg Sports Traumatol Arthrosc 2011;19(8):1326-1333

11 Fernandes JL, Viana SL, Mendonça JL, et al. Mucoid degeneration of the anterior cruciate ligament: magnetic resonance imaging findings of an underdiagnosed entity. Acta Radiol 2008;49(1):75-79

12 Lintz F, Pujol N, Dejour D, Boisrenoult P, Beaufils P. Anterior cruciate ligament mucoid degeneration: selecting the best treatment option. Orthop Traumatol Surg Res 2010;96(4):400-406

13 Narvekar A, Gajjar S. Mucoid degeneration of the anterior cruciate ligament. Arthroscopy 2004;20(2):141-146

14 Hsu CJ, Wang SC, Fong YC, Huang CY, Chiang IP, Hsu HC. Mucoid degeneration of the anterior cruciate ligament. J Chin Med Assoc 2006;69(9):449-452

15 Cha JH, Lee SH, Shin MJ, Choi BK, Bin SI. Relationship between mucoid hypertrophy of the anterior cruciate ligament (ACL) and morphologic change of the intercondylar notch: MRI and arthroscopy correlation. Skeletal Radiol 2008;37(9):821-826

16 Kim TH, Lee DH, Lee SH, Kim JM, Kim CW, Bin SI. Arthroscopic treatment of mucoid hypertrophy of the anterior cruciate ligament. Arthroscopy 2008;24(6):642-649

17 Muir P, Schamberger GM, Manley PA, Hao Z. Localization of cathepsin $\mathrm{K}$ and tartrate-resistant acid phosphatase in synovium and cranial cruciate ligament in dogs with cruciate disease. Vet Surg 2005;34(3):239-246

18 Barrett JG, Hao Z, Graf BK, Kaplan LD, Heiner JP, Muir P. Inflammatory changes in ruptured canine cranial and human anterior cruciate ligaments. Am J Vet Res 2005;66(12):2073-2080

19 Akiyama T, Bouillet P, Miyazaki T, et al. Regulation of osteoclast apoptosis by ubiquitylation of proapoptotic BH3-only Bcl-2 family member Bim. EMBO J 2003;22(24):6653-6664

20 Akiyama T, Dass CR, Shinoda Y, Kawano H, Tanaka S, Choong PF. Systemic RANK-Fc protein therapy is efficacious against primary osteosarcoma growth in a murine model via activity against osteoclasts. J Pharm Pharmacol 2010;62(4):470-476

21 Akiyama T, Choong PF, Dass CR. RANK-Fc inhibits malignancy via inhibiting ERK activation and evoking caspase-3-mediated anoikis in human osteosarcoma cells. Clin Exp Metastasis 2010;27(4): 207-215 